\title{
Systematic literature review on novel corona virus SARS-CoV-2: a threat to human era
}

\author{
Dinesh Kumar Rajendran ${ }^{1}$ - Varthini Rajagopal ${ }^{2}$ (1) S. Alagumanian ${ }^{3} \cdot$ \\ T. Santhosh Kumar ${ }^{4}$ - S. P. Sathiya Prabhakaran ${ }^{5}$ - Dharun Kasilingam ${ }^{6}$
}

Received: 4 April 2020/ Accepted: 24 May 2020/Published online: 11 June 2020

(C) Indian Virological Society 2020

\begin{abstract}
Severe acute respiratory syndrome coronavirus 2 (SARS-CoV-2) is the seventh-generation coronavirus family causing viral pandemic coronavirus disease (COVID-19) across globe affecting millions of people. The objectives of this study are to (1) identify the major research themes in COVID-19 literature, (2) determine the origin, symptoms and modes of transmission of COVID, (3) recommend the intervention and mitigation strategies adopted by the Governments globally against the spread of COVID-19 and the traumatization among the public? and (4) study the possible drugs/treatment plans against COVID-19. A systematic literature review and comprehensive analysis of 38 research articles on COVID-19 are conducted. An integrated Research focus parallel-ship network and keyword co-occurrence analysis are carried out to visualize the three research concepts in COVID-19 literature. Some of our observations include: (1) as SARS-
\end{abstract}

Electronic supplementary material The online version of this article (https://doi.org/10.1007/s13337-020-00604-z) contains supplementary material, which is available to authorized users.

Varthini Rajagopal

varthini.jaku@gmail.com

1 Department of Mechanical Engineering, National Institute of Technology Goa, Goa, India

2 Department of Mechanical Engineering, Government College of Engineering Srirangam, Tiruchirappalli, Tamilnadu, India

3 Department of Botany, H.H. The Rajah's College, Pudukkottai, Tamilnadu, India

4 Department of Pediatrics, JIPMER, Pondicherry, India

5 Department of Energy and Environment Engineering, NITTiruchirappalli, Tiruchirappalli, India

6 Digital Platform and Strategies, MICA The School of Ideas, Ahmedabad, India
CoV-2's RNA matches $\sim 96 \%$ to SARS-CoV, it is assumed to be transmitted from the bats. (2) The common symptoms are high fever, dry cough, fatigue, sputum production, shortness of breath, diarrhoea etc. (3) A lockdown across 180 affected counties for more than a month with social-distancing and the precautions taken in SARS and MERS are recommended by the Governments. (4) Researchers' claim that nutrition and immunity enhancers and treatment plans such as arbidol, lopinavir/ritonavir, convalescent plasma and mesenchymal stem cells and drugs including remdesivir, hydroxychloroquine, azithromycin and favipiravir are effective against COVID-19. This complied report serves as guide to help the administrators, researchers and the medical officers to adopt recommended intervention strategies and the optimal treatment/drug against COVID-19.

Keywords SARS-CoV-2 - Origin · Symptoms · Drugs · Interventions $\cdot$ Network analysis

\section{Introduction}

Coronavirus $(\mathrm{CoV})$ is clustered under the viral family group that causes disease in mammals and birds. A pandemic novel coronavirus was named as "Corona Virus Disease 2019" (2019-nCoV) by World Health Organization (WHO) in Geneva, Switzerland [28, 47, 49]. This is the deadly third-generation virus in Corona family preceded by Middle East Respiratory Syndrome (MERS) in 2012 and Severe Acute Respiratory Syndrome (SARS) in 2003. After Rhinoviruses, Coronaviruses are ranked as the main cause of the commonplace cold without triggering any sickness [40]. In 1937, the primary coronavirus was located in bats, rarely affecting humans and mostly 
circulating among animals like bats, camels, and cats. Later, they mutated to contaminate rats, cattle, pigs, mice, cats, dogs, horses, and turkeys. The first human coronavirus, known in the sixties, infected the nasal cavities of humans resulting in common cough and cold. Symptoms in different species includes: higher breathing tract ailment in chickens, diarrhoea in cows and pigs [46], dry cough, tiredness, fever, sore throat, aches and pains, shortness of breath, runny nose and diarrhoea in humans $[12,45]$.

Coronaviruses establish the subfamily Orthocoronavininae inside the family Coronavidae, and belong to the order Nidovirales, and the realm Riboviria [31]. They are encircled and the most acknowledged RNA virus until date with a nucleocapsid and a superb-experience single-stranded RNA genome with the genome length ranging from $\sim 27$ to 34 kilobases [1, 39]. A two-dimensional view of Corona beneath a transmission electron microscopy reveals a characteristic look of "paying homage to a crown" around the virions. [47] This lead to naming the virus "Corona", meaning "crown" or "halo" in Latin. As its RNA patten is closer to SARS, the 2019 Coronavirus is renamed as SARS-CoV-2 pandemic. In 2003, SARS-CoV killed almost $10 \%$ of total affected patients (8429) across 29 international locations. MERS$\mathrm{CoV}$ was found to be even more lethal with a mortality rate of $30 \%$ of the infected patients [35]. Comparatively, the mortality rate of SARS-CoV-2 is very less $(2.9 \%)$ albeit resulting in a massive outbreak with 491,271 confirmed cases and 22,165 deaths across 198 countries, as on 26th March 2020. This exponential death rate across different regions might not be as serious as Black death which killed around 75 million people in the fourteenth century and Influenza which resulted in 50 million death in 1918 [1], but if proper precautions are not taken there might be a greater chance of SARS-CoV-2 becoming the most devasting pandemic disease of the twenty-first century.

In order to overcome the above disastrous scenario and serve as an aid to the healthcare researchers and the Government administrators, our work presents a systematic literature review (SLR) and a comprehensive analysis of 38 research articles on Coronaviruses 2019 (COVID-19). An integrated Research Focus Parallel-ship Network (RFPN) and Keyword Co-Occurrence Network (KCON) is formulated to visualize the major research themes in COVID-19 literature. The rest of this paper is organized as follows: "Systematic literature review" section presents the SLR and co-occurrence network analysis. The "A Comprehensive analysis of reviewed papers" section reports a comprehensive analysis of the reviewed papers in consideration with the origin, mutational outbreak and the modes of transmission of SARS-CoV-2, the intervention/control and precaution measures taken by the different counties across the globe against the exponential pandemic growth, and the updates on the drugs/treatment plans/clinical vaccines against COVID-19. Finally, the conclusions are highlighted in "Conclusions" section.

\section{Systematic literature review}

Following [38], a three-phase SLR methodology was adapted. Phase I involves the formulation of the research questions and our work focuses on answering the research question: "What are the major research streams in COVID19?"

Then, Phase II is carried out where the articles for the study are selected and filtered. The articles are accessed from the Web of Science database using the keywords "COVID", "CORONA", "SARS", "WUHAN VIRUS", "WUHAN PNEUMONIA" or "nCoV". A total of 156 papers published during 2019-2020 were collected and were subjected to screening. Within this initial sample, short communications and letters to editors are filtered. Then, the title, abstract, and keywords of each paper were checked for relevance and subsequently, 38 papers were found suitable for the study. Table 1 shows the top distribution of the reviewed papers by the journal.

Using the screened sample of 38 papers, Phase-III is performed which involves data extraction and co-occurrence network $(\mathrm{CON})$ analysis followed by a comprehensive reporting. CON analysis is a bibliometric tool useful for investigating, mapping, and visualizing the major research concepts in a particular scientific field. CON maps the author keywords to construct a network that represents the intellectual content of the research field such as cognitive themes and their interrelations using cluster analysis and network analysis [5]. Cluster analysis is carried out using RFPN and the research streams of each cluster are identified using the KCON. The procedure of RFPN and KCON is adapted from [38] which involves (1) title and author keywords retrieval, (2) Revision and statistical analysis, (3) Visualization and clustering of RFPN, and (4) Research themes identification using KCON.

Table 1 Distribution of reviewed papers by journal

\begin{tabular}{ll}
\hline Journal & No. of papers \\
\hline 3 & International Journal of Infectious Diseases \\
3 & Journal of Medical Virology \\
2 & Engineering \\
2 & Journal of Korean Medical Science \\
2 & Journal of Advanced Research \\
2 & Journal of Microbiology, Immunology And Infection \\
2 & Travel Medicine And Infectious Disease \\
\hline
\end{tabular}


The title and author keywords are retrieved from the 38 papers using BibExcel [17]. Following [24], each word in the title and author keywords (collectively known as an actor) are standardized as different authors use different words to describe the same meaning. The common terms like "COVID-19", "CORONA", "nCoV", "Wuhan" and "China" are excluded in the RFPN analysis as they will result in a lower centrality measure for the actors that represent the core research concept in each cluster. The actors with a single frequency are excluded as they cannot be mapped in CON. In all, 60 actors are identified and the top 15 keywords by frequency are listed in Table 2.

In RFPN, each paper denotes a node and an edge is formed between two papers if they have at least one common actor amongst them. The number of common actors between two papers represents the edge weight between them. We use the open-source software Gephi to map and visualize the actor co-occurrence among the reviewed papers [3]. Based on the data extracted using BibExcel, a raw RFPN network consisting of 38 nodes and 135 edges is created in Gephi with a random positioning of the nodes. To identify and eliminate the outliers i.e. papers with no connection to the network, the Force Atlas Layout is used which moves the highly connected nodes to the centre of the network and the least connected nodes away from the centre. Figure $1 \mathrm{a}-\mathrm{c}$ show the raw network comprising the 38 nodes, the force layout of the raw network with and without the outliers (3 papers) respectively.

Next, we group the 35 nodes into communities based on the modularity index, where each community represents a set of densely connected papers with sparse connections to the other clusters [38]. For this purpose, we use the default

Table 2 Top 15 actors with frequency
Louvain clustering algorithm to partition a network into the optimal number of communities that maximizes the modularity index [23]. Figure 2 shows the RFPN clustered into 3 modules each representing a specific research concept and the number within each node denotes the paper id (see "Online Appendix"). The modularity index of the RFPN is 0.603 , which indicates that an optimal number of clusters is identified [34]. The lead nodes of each cluster have higher values of Eigenvector Centrality (EVC) measure which varies from 0 to 1 and denotes how well connected a node is within the network [9]. The node sizes shown in Fig. 2 are proportional to their EVC scores. Table 3 shows the leading 5 articles based on the EVC value in each community.

From Fig. 2, it is clear that Cluster 3 is the highly studied research stream in the COVID-19 literature and includes 13 papers. From Table 3, we can also observe that clusters 3 includes papers with a higher EVC compared to other clusters. Based on the position in the RFPN and EVC, the influence of a paper in the cluster can be determined. Accordingly, the core research theme of a cluster is determined by the papers with a higher EVC in that cluster.

Next, KCON is constructed using Gephi to determine the research themes in each cluster. KCON represents each actor as a node and an edge link is formed between two actors if they are listed in the same paper. The number of common papers listing both the actors determines the edge weight between them. The actors of the papers in each cluster are extracted and the actor co-occurrence array in a .net format is generated using BibExcel. Using the .net file, a raw network with randomly positioned actors is developed using Gephi. The Force Layout algorithm is then applied to move the most connected actors to the centre and the less connected actors away from the centre of the network.

Figures 3, 4 and 5 represent the KCON of clusters 1, 2, and 3 respectively. The sizes of the actors are proportional to their EVC. The actors with higher EVC and central to the network with thicker edges represent the major research theme in that cluster. For example, the core keywords of cluster 1 are 'Symptoms', 'Transmission', 'Origin', and 'Spread'.

Accordingly, the major research concepts addressed in each cluster are identified as shown in Table 4.

\section{A comprehensive analysis of reviewed papers}

Based on the findings of the $\mathrm{CON}$ analyses, the research questions are expanded to include the following: (1) what are the origin, symptoms and modes of transmission of COVID-19? (2) what are the possible intervention and mitigation strategies that can be adopted globally against 

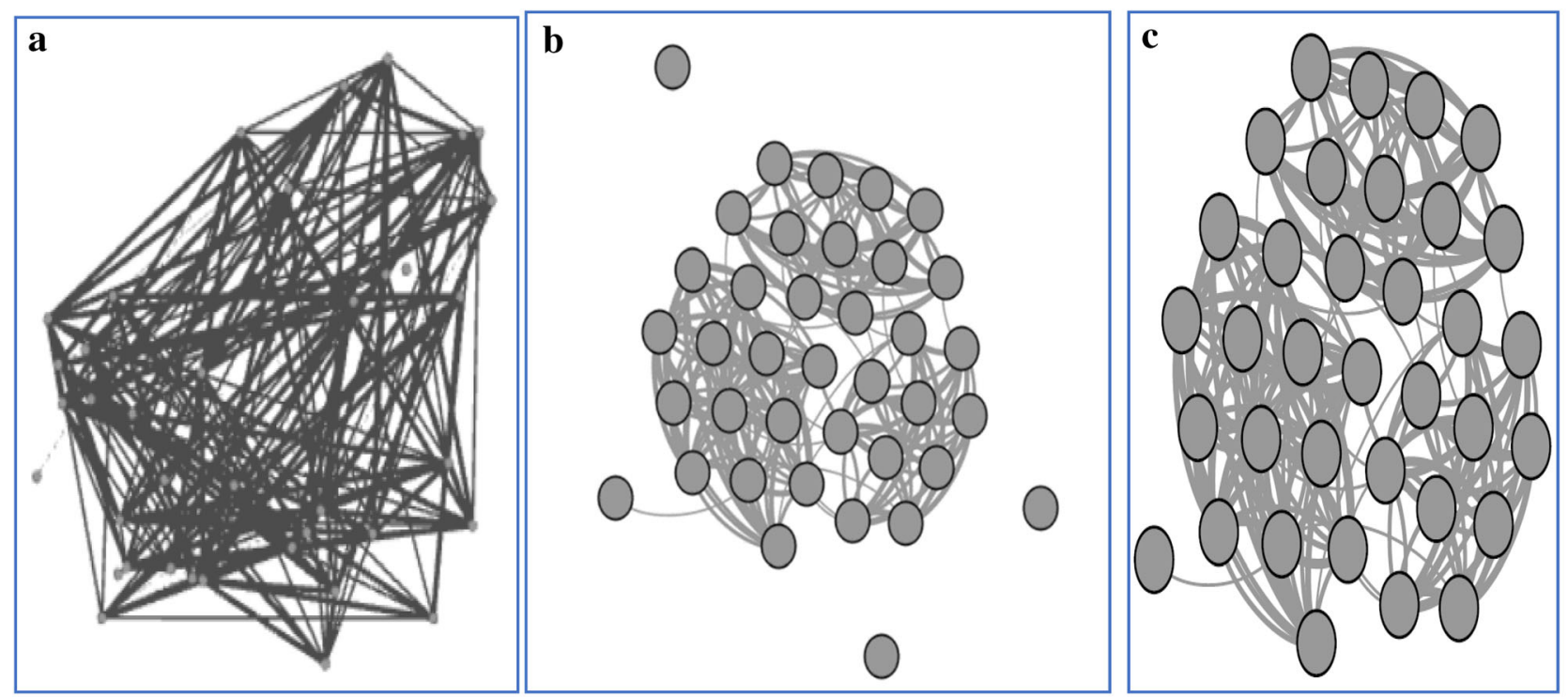

(a) Initial network (38 nodes) (b) Network with outliers (c) Final network (35 nodes)

Fig. 1 RFPN network

Fig. 2 Clustered RFPN with node size proportional to its EVC

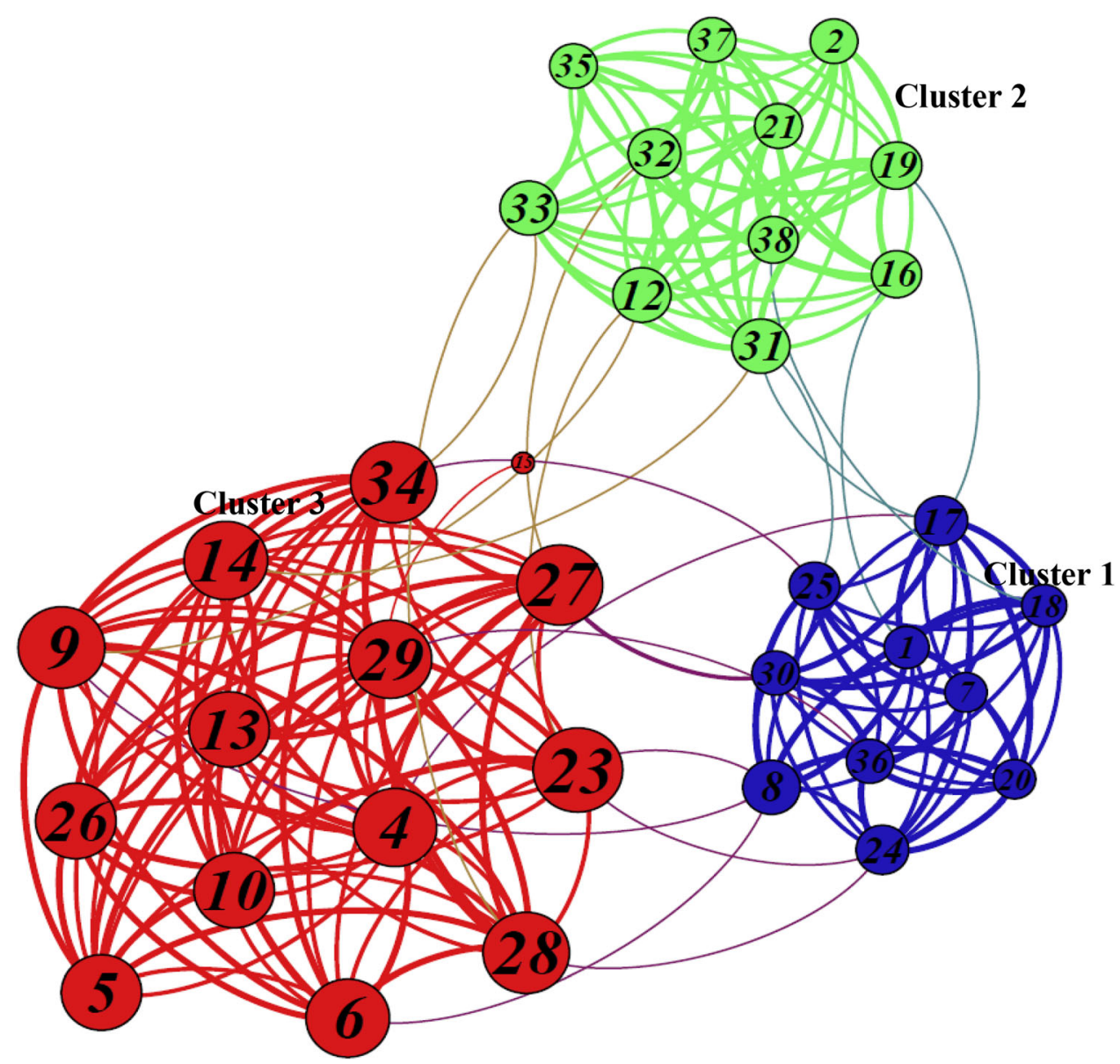


Table 3 Leading 5 articles in RFPN clusters based on EVC

\begin{tabular}{|c|c|c|c|c|c|c|}
\hline \multirow[t]{2}{*}{ Rank } & \multicolumn{2}{|l|}{ Cluster 1} & \multicolumn{2}{|l|}{ Cluster 2} & \multicolumn{2}{|l|}{ Cluster 3} \\
\hline & Article ID & EVC & Article ID & EVC & Article ID & EVC \\
\hline 1 & [8] & 0.555 & [32] & 0.563 & [9] & 0.961 \\
\hline 2 & [16] & 0.494 & [12] & 0.561 & [28] & 0.956 \\
\hline 3 & [24] & 0.488 & [34] & 0.559 & [35] & 0.954 \\
\hline 4 & [25] & 0.461 & [33] & 0.488 & [27] & 0.945 \\
\hline 5 & {$[30]$} & 0.414 & [18] & 0.459 & [19] & 0.918 \\
\hline
\end{tabular}

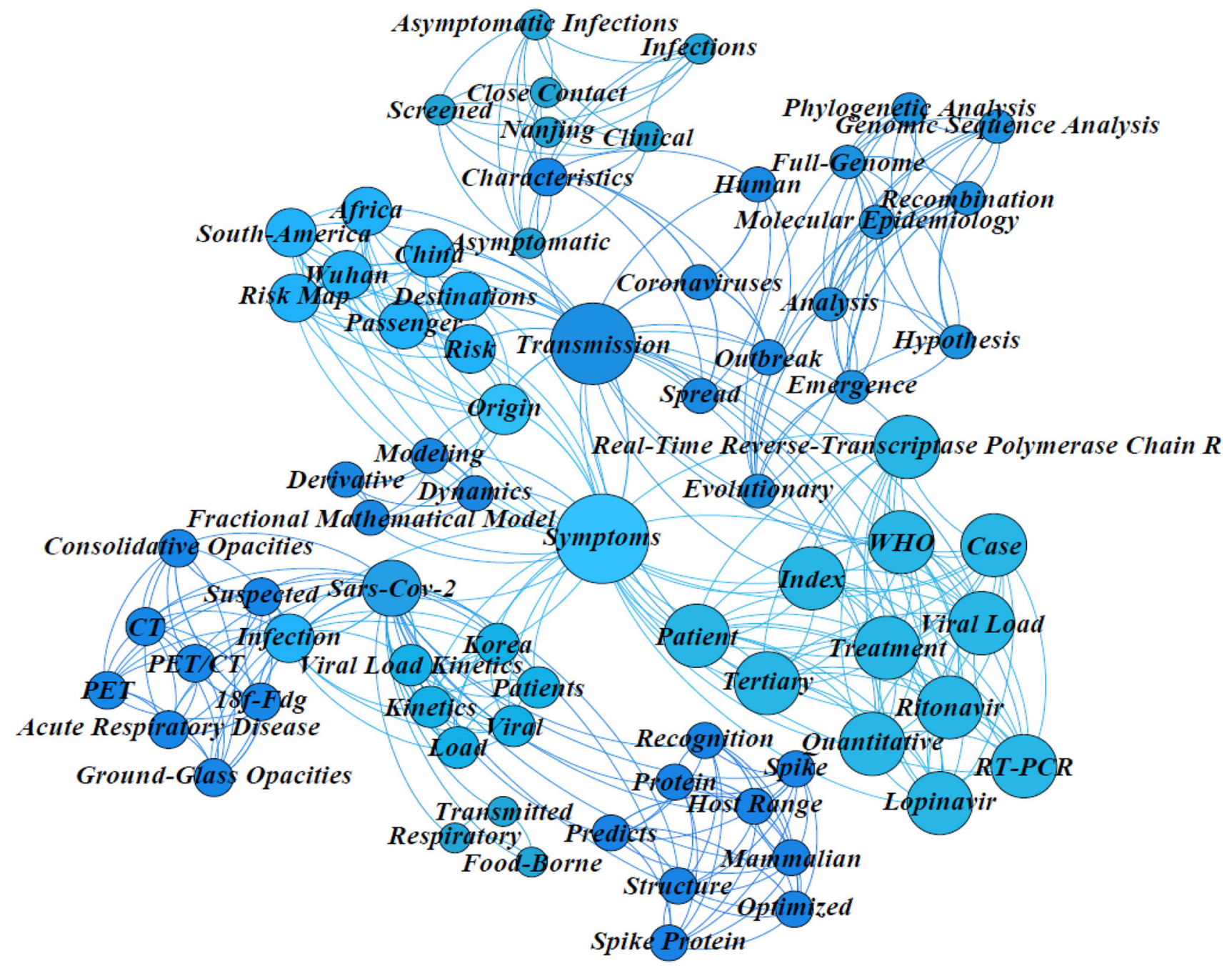

Fig. 3 KCON of cluster 1: origin, symptoms and modes of transmission of COVID-19

the spread of COVID-19 and traumatization among the public? and (3) what are the possible drugs/treatment plans proposed against COVID? To answer the above research questions, the 38 papers are further comprehensively analysed. 


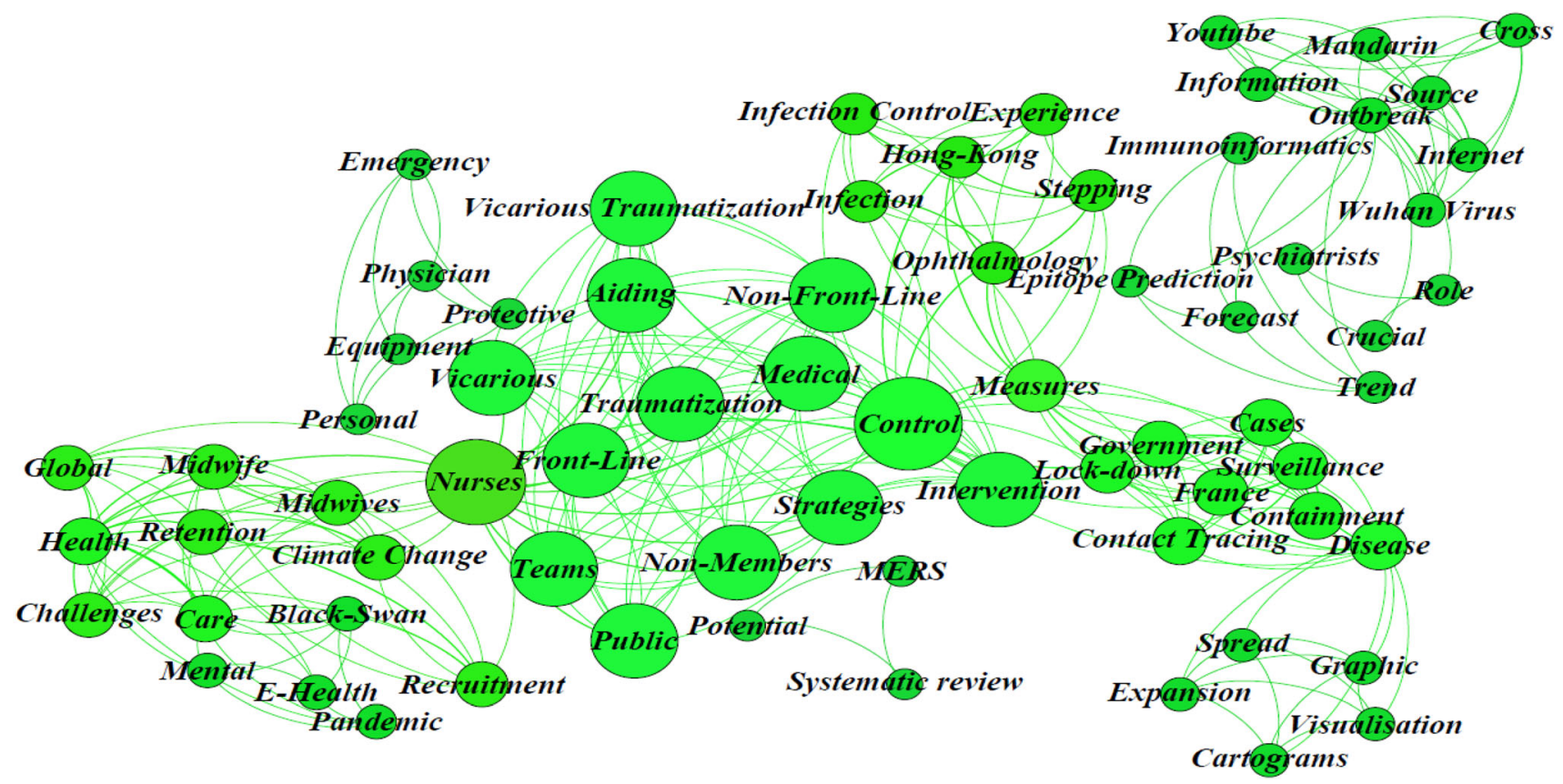

Fig. 4 KCON of cluster 2: intervention and mitigation strategies against the spread of COVID-19 and traumatization among the public

Cluster 1: origin, symptoms and modes of transmission of COVID

Genetical morphology, classifications and replication mechanism

Morphology Mostly viruses have either DNA or RNA genetic materials. SARS-CoV-2 belongs to a large family of RNA viruses and has a single stand RNA structured genome with 32 kilobases length [41]. This is found to be the largest RNA virus genome known to mankind. SARS$\mathrm{CoV}-2$ has a unique feature i.e., the recombination frequency of positive-strand RNA is very high. When the host is infected with multiple coronaviruses, they promiscuously combine genetic information from different sources resulting in a high mutation rate which creates major confusions during diagnostics and vaccine generation [19]. Morphology of Corona Virus as adopted from [28] is shown in Fig. 6.

Classifications of coronavirus Coronavirus is majorly classified into four types which are named after the Greek letters alpha $(\alpha)$, beta $(\beta)$, delta $(\delta)$ and gamma $(\gamma)$. In the above four classifications, the categories that infect human beings are alpha and beta coronaviruses. These viruses spread through the aerosol and airborne modes of transmission which mark a responsibility of about $10-30 \%$ of colds occurring worldwide. Coronavirus was not deemed dangerous until it underwent mutational change resulting in upper respiratory infections and pneumonia [31]. Till date the researchers have found seven human coronaviruses (HCoVs) which are listed as follows: SARS, MERS, SARS-CoV-2, HCoV-229E, HCoV-OC43, HCoV $-N L 63, H C o V-H K U 1$ [39]. 2019-nCoV have mutated from aerosol to airborne with a communal distance of 4-5 feet through droplet nuclei mixed with high humid air, causing respiratory disease in children and aged population.

Replication mechanism Generally, the past reported RNA virus genomes which infected mankind contains a single open reading frame (ORF) and the ORP is converted into single polyprotein. This polyprotein is further catalytically sliced into shorter functional viral proteins. However, the replication mechanism of Coronaviruses is found to have an unusual pattern involving a 2-step replication as shown in Fig. 7 [31]. A single Coronavirus holds more than 10 separate ORFs enabling the virus to grow and spread at an uncontrollable rate [39]. The biggest ORFs is then translated by ribosomes in Step 1 and on comparing with an RNA viral genome, they are twice in size. Then, the enzymes are encoded by replicate gene using other genomes as template producing messenger RNA molecules which is further transformed into structural protein that acts as building block of new viral particles in Step 2 [16].

\section{Origin and primary modes of transmission}

SARS-CoV in 2003 and MERS-CoV in 2012 was found to be originated from bat and dromedary camel respectively. 


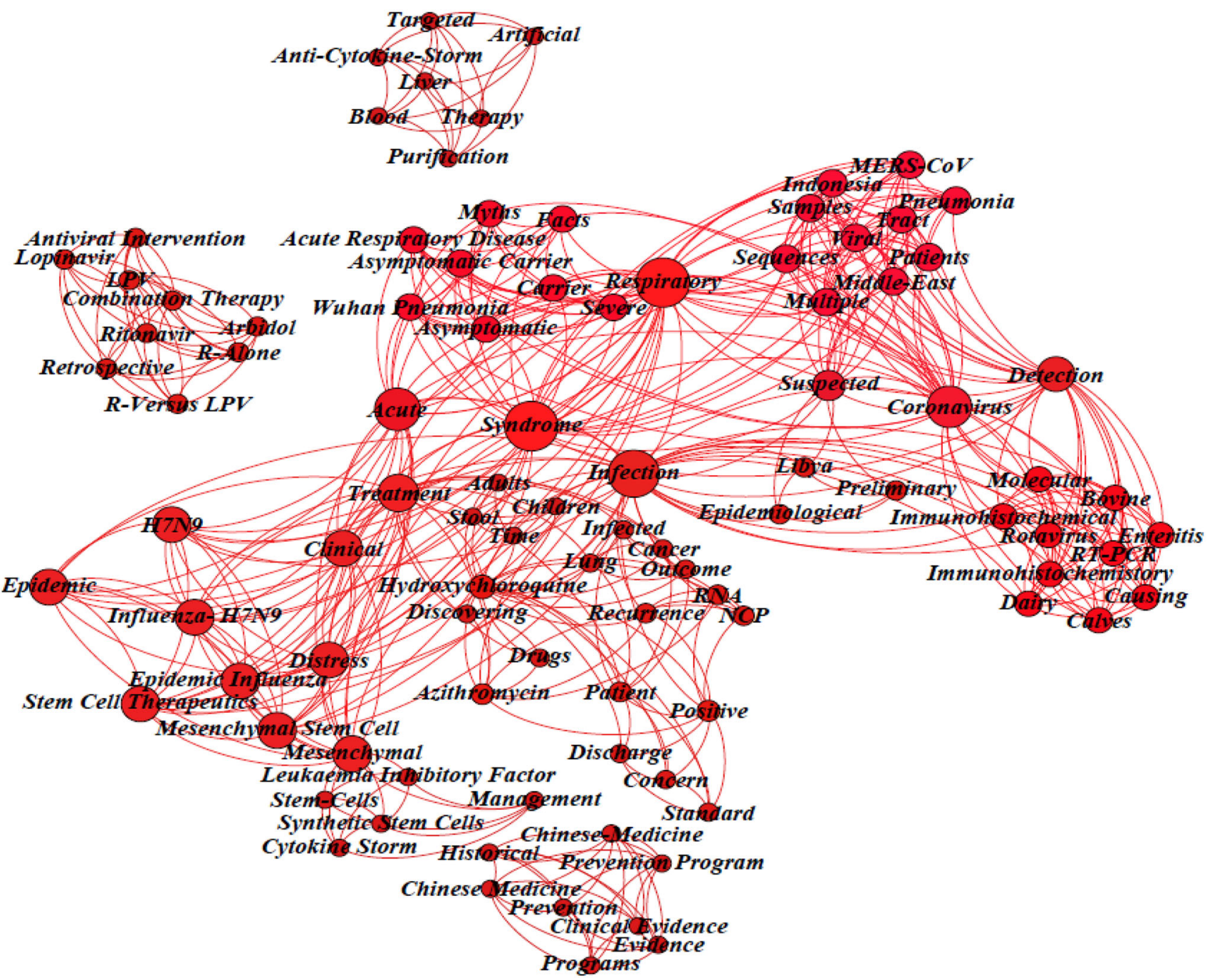

Fig. $5 \mathrm{KCON}$ of cluster 3: drugs and treatment plans of COVID-19

Table 4 Major research concepts in COVID-19 identified using KCON

\begin{tabular}{lll}
\hline Cluster & No. of papers & Research area \\
\hline 1 & 10 & Origin, symptoms and modes of transmission of COVID-19 \\
2 & 11 & Intervention and mitigation strategies against the spread of COVID-19 and traumatization among the public \\
3 & 14 & Drugs and treatment plans of COVID-19 \\
\hline
\end{tabular}

In this series, SARS-CoV-2 which has $\sim 96 \%$ similarity to SARS-CoV is also assumed to be originated from the bats and transmitted through animal-human contact in Wuhan meat market, Hubei province, China. However, because of mutation change, it had acquired the capacity to transfer from human to human [40]. As on 26th March 2020, 4,91,579 cases have been reported across 196 nations with 17,234 death and 1,03,732 recuperated cases.
Jalava [20] highlighted that the introductory COVID-19 cases might have occurred due to Zoonotic mode of transmission. Because of the restricted initial human-tohuman spread, the timely grouping of the elderly people in Huanan Seafood Market, Wuhan, China, and the association between bat coronavirus and SARS-CoV-2, Zoonotic infection might have resulted. Further, the SARS-COV-2 epidemic might have originated from the other unusually large pollutants in the seafood industry varying from 


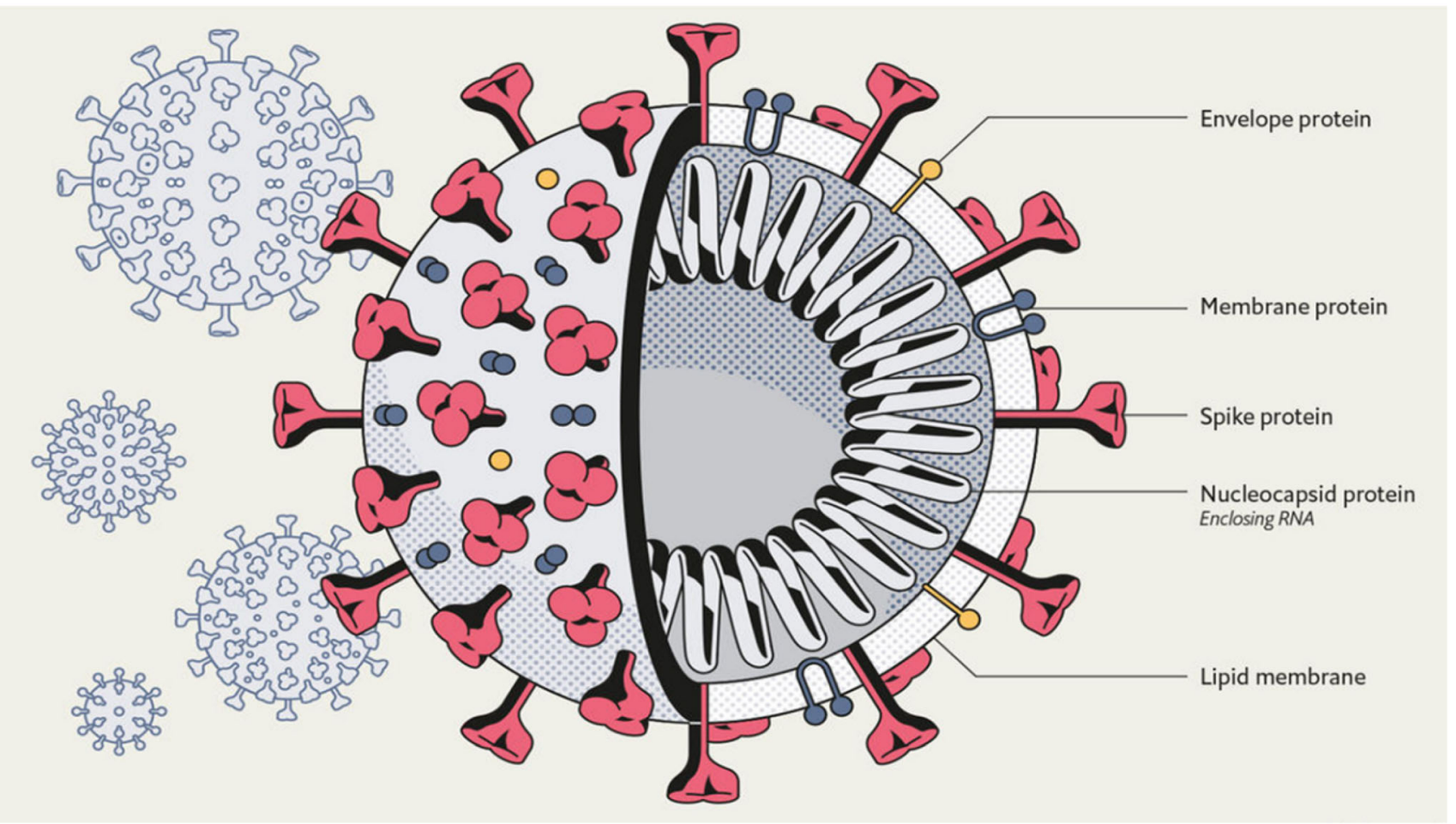

Fig. 6 Morphology of corona virus. Source: [28]

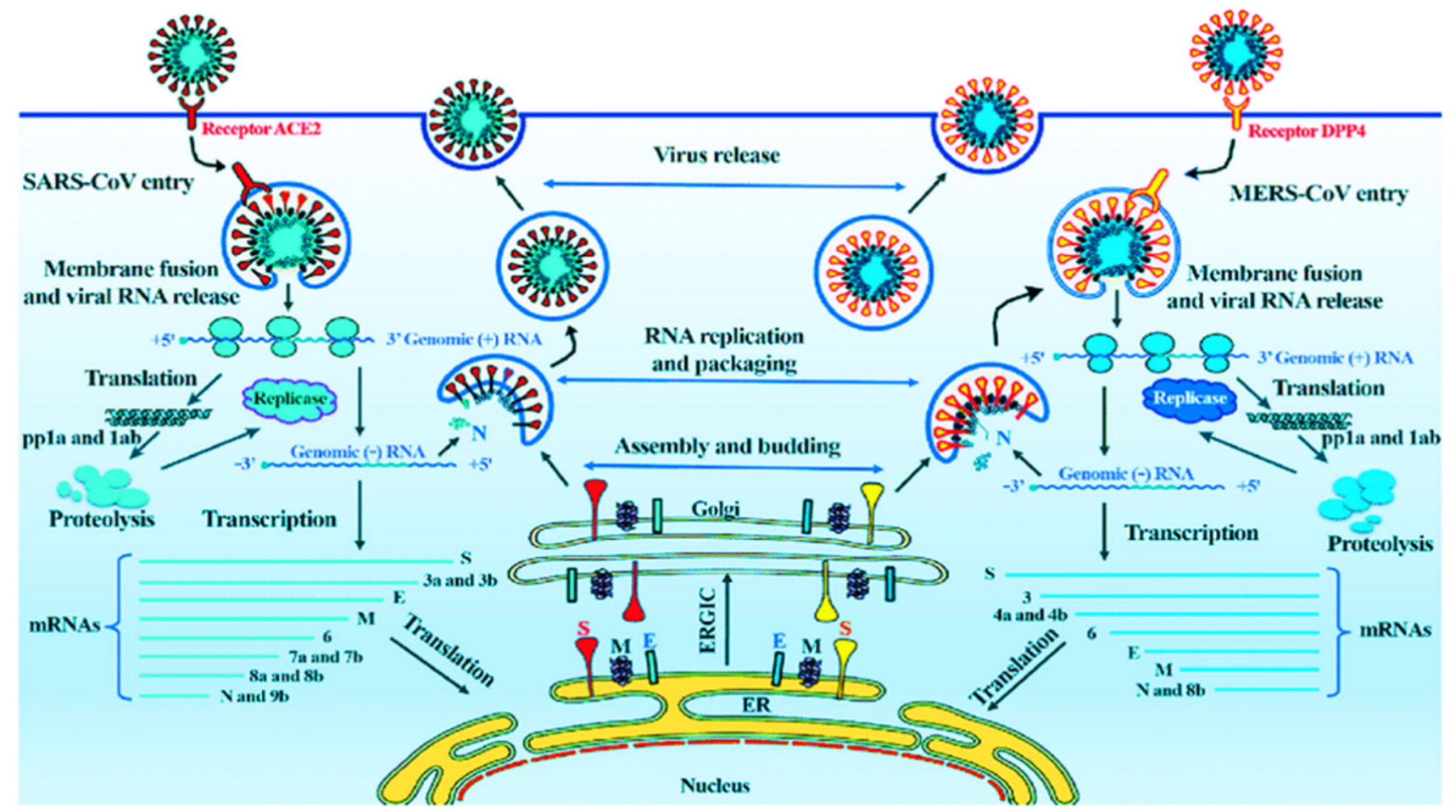

Fig. 7 Replication mechanism of coronaviruses (Source: [31])

seafood bins to air pollution and from livestock or rats. Haider et al. [15] estimated a transmission risk index of COVID-19 based on the number of passengers to destination countries, weighed by the number of recorded instances in the departed cities, reported by the WHO. Based on the developed risk index, they ranked each nation 
in four quantiles threat level (4th quantile represents the greatest risk and 1st quantile denotes the lowest risk) covering 388,287 travellers connected to 1297 airports in 168 countries all over the worldwide. As per their risk rating system, the countries under study reported at least one COVID-19 case as of 5 February 2020, and the number of countries in the fourth, third, second and first quantile of the threat level were 24, 2, 1 and none respectively. Except China, higher-risk countries of 2019-nCoV transmission included: Thailand, Canada, Malaysia, Cambodia, the USA, Germany, UK, Italy France, Russia, and Australia, each reporting a minimum of one case. In Africa and South America, the risk of infection is very small with Brazil, South Africa, Mauritius, Ethiopia and Egypt displaying a higher danger of transmission. Improved public safety response by early event identification, suspicious accidents monitoring, contract tracking and improved airport security, public awareness and safety staff caution are some of the intervention techniques proposed against the spread to other territories.

\section{Symptoms and secondary modes of transmission}

Qin et al. [37] have reported that COVID-19 has pulmonary tropism resulting in peripheral ground-glass opacities and/or lung consolidations in more than two pulmonary lobes. They recommended the usage of ${ }^{18} \mathrm{~F}$ FDG PET/CT imaging technique to examine the lung lesions and lymph node which are characterized as COVID-19 symptoms.

Lim et al. [27] discussed the spread of COVID-19 in South Korea through secondary transmission from the people who travelled from China. A textile worker, who caused one and three cases of secondary and tertiary transmissions respectively, was observed to have moderate respiratory signs and irregular body temperature during hospitalization accompanied by pneumonia symptoms on the chest computerized tomography (CT) examination on day 6 of admission.

$\mathrm{Hu}$ et al. [18] exposed the dissemination potential of asymptomatic COVID-19 virus transmission by examining the medical characteristics of 24 cases of asymptomatic patients who acquired the infection from near-contact. During the screening, none exhibited any oblivious symptoms. But later they were hospitalized and $20.8 \%$ evolved symptoms (cough, fatigue, fever, and many others), $50.0 \%$ showed floor-glass chest in CT images and $20.8 \%$ exhibited stripe shadowing in the lungs. The remaining cases, who showed no symptom with normal CT image, were more youthful and out of 24 instances none developed extreme COVID-19 pneumonia nor demised. This work also reported that communal period varies from 9.5 days in symptomatic patients to 21 days among the asymptomatic patients. Continuous Virus Nucleic Acid tests and isolation were recommended for the patients to be discharged.

Paraskevis et al. [36] classified the genetic links of 2019-nCoV and checked for putative hybridization within the genus of Sarbecovirus. Hypothetical recombination was explored by using RDP4 and Simplot V3.5.1 and discordant phylogenetic clustering in person genomic fragments was validated using phylogenetic analysis via Maximum likelihood and Bayesian strategies. The results indicate that the 2019-nCoV is strongly linked to BatCoV Ratg13 code in the genome (code similarity 96.3\%); though latter will not result in epidemic as they lack the precise mutation. However, the evidence that 2019-nCoV arose from bats was quite conceivable with $\mathrm{nCoV}$ being non-mosaic in nearly half of its genome of a unique lineage inside the Betacoronavirus. Such genomic attributes and their possible interaction with the properties of viruses and virulence in humans require more focus.

Luan et al. [29] highlighted that ACE2 is a processor for both the coronaviruses SARS-CoV-2 and SARS-CoV and by evaluating the main residues of ACE2 for $S$ Protein Recognition, the probable host range of $\mathrm{nCoV}$ can be determined. They observed that pets (dog and cat) and Pangolin and Circetidae mammals retained much of the essential residues for connection with $S$ Protein from SARS-CoV and nCoV. The communication between cat/dog/pangolin/Chinese hamster ACE2 and S Protein from SARS-CoV/nCoV was modelled by homology simulation. They also identified that N82 ACE2 had a greater interaction with SARS-CoV-2 S Protein than M82 in human ACE2 and provided an understanding into the host repertoire of SARS-CoV-2 and highlighted a new approach for developing and optimizing ACE2 for SARS-Cov-2 infection.

Kim et al. [21] focused on Viral Load Kinetics of 2 reported COVID-19 cases with mild to medium illness in Korea. Their results show that COVID-19 exhibits a unique and distinct Viral Load Kinetics than that of the other previously reported Coronaviruses.

Max Roser and Ortiz-Ospina [32], described the symptoms of COVID-19 based on the data of 55,924 laboratory confirmed cases of $\mathrm{nCoV}$ in China, provided by WHO as on February 20, 2020. Figure 8, adopted from Roser maps the symptoms of COVID-19.

\section{Cluster 2: intervention and mitigation strategies against the spread of COVID and traumatization among the public}

In this section various strategies taken by government, health sector, researcher and general public against the spread of COVID-19 is discussed. Stoecklin et al. [4] reported the intervention strategies taken by France. 


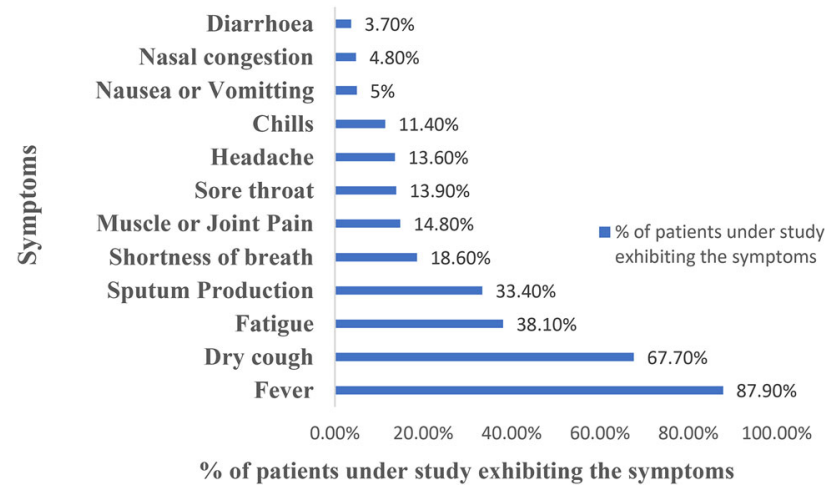

Fig. 8 Symptoms of coronavirus. Source: [32]

Starting from 10th January 2020, strengthened surveillance with effective collaboration among the stakeholders was implemented to identify the imported cases from other countries and to control secondary transmission risk. A follow-up procedure with necessary control measures was also defined at three risk levels.

Catton [6], related the global challenges faced due to COVID-19 to global warming. The challenges faced by healthcare professionals were highlighted. Adequate number hospital nurses were recommended to reserve as buffer while the remaining nurses should be employed on rotation basis.

$\mathrm{Li}$ and Feng [25], discussed the significant identification of Cytotoxic T Lymphocyte (CTL) and B cell epitopes in nCoV Surface Glycoprotein by Immunoinformatics approach. This article uses molecular dynamics simulations to study the interactions between major histocompatibility complex (MHC) and the identified CTL epitopes in China. The viral surface glycoprotein contained 5 CTL epitopes, 3 sequential $\mathrm{b}$ cell epitopes and 5 discontinuous b cell epitopes. MHC class I peptide-binding grooves were found to be attached to CTL epitopes with persistent hydrogen bonds and salt bridge anchors. This highlights their capacity to induce immune responses and hence to serve as potential candidates for the production of $\mathrm{nCoV}$ vaccines.

Lai et al. [22] explains about their local experience in minimising COVID-19 through ophthalmological control measures for both patients and health care workers. They implemented a three-level system of administrative management measures. The first level includes reducing the patient presence by sending text messages with an inquiry contact number of clinicians for rescheduling the medications, mitigating the cross-infection of COVID-19 by establishing a triage system to segregate patients with symptoms and travel history and preventing non-contact tonometry and procedures under general anaesthesia that generates micro-aerosol. Further, they avoided nasal endoscopy that provokes sneezing and produces droplets. They also suspended all elective clinical treatments and the staffs were given training on control measures. Secondly, environmental safety measure such as prevention of the spread of COVID-19 droplets, implementation of security covers on slit lights, the regular disinfection of appliances and the provision of eye protection to workers were introduced. Thirdly, protection measures such as uniform masking, hand grooming and correct usage of Personal Protective Equipment (PPE) were encouraged.

Banerjee [2] used a total of 214 general public and 526 patients, to analyse the vicaria trauma scores using a smartphone app-based assessment to tackle the psychological stress of the working citizens. The findings indicate that the clinical burden of non-frontline nurses was greater than that of frontline nurses, and the general public's vicaria distress ratings were slightly higher than that of the patients. Wind et al. [43] and Li et al. [26] also highlights that the lack of proper awareness and precautions about COVID-19 is increasing the stress among the public.

Gao et al. [14] addressed the WHO's progress on the transparency and the open-access to the disease data graphs. As choropleth map writers have suggested, cartograms are the best tool to represent both the growth and the distribution of COVID-19. Cartograms of six circular variations were designed comprising details on the verified cases every 48 hours. Such styles of projects, communicate knowledge more intuitively and effectively, thereby encouraging greater public engagement in the battle against COVID-19.

Yan et al. [44] discuss the analysis of YouTube video as a tool to hinder COVID-19. 72 videos in English and 42 in Mandarin about COVID-19 were reviewed. The videos were classified as useful, misleading or news-based. Kappa coefficient was used to evaluate the Inter-observer agreement. This analysis showed that YouTube viewership during $2019 \mathrm{n}-\mathrm{CoV}$ outbreak is higher than the previous outbreaks. They also advocated that passing on the accurate and authentic information to general public through YouTube by controlling or prioritising video delivery can help the general public to fight against COVID-19.

\section{Cluster 3: drugs and treatment plans of COVID-19}

In this cluster, the various drug and treatment plans published by different researchers to fight against COVID-19 are compiled. Deng et al. [11] reported a treatment plan based on Arbidol and Lopinavir/Ritonavir (LPV/R). Clinical observation for a period of 5-21 days between Jan 17, 2020, to Feb 13, 2020, was carried out for COVID-19 confirmed patients without invasive ventilation. The patients were administrated Oral Arbidol and LPV/R in combination and monotherapy groups. Chest CT, taken to check pneumonia on Day 7 and Day 14, exhibited a drastic improvement on the patients in the combined group with 
Arbidol and LPV/R. 94\% success rate was observed in the combination group than the monotherapy group $(30 \%$ success rate).

Lai et al. [22] and Zhang et al. [48] reported different forms of COVID-19 such as Pneumonia, Acute Respiratory Disease and Asymptomatic Carrier State. They observed that the highest infection rate is with adults followed by neonates, elderly patients and, children. Ground-Glass Visibility Bilateral interaction was observed in the Chest Imaging of the infected patients. They advocated the usage of drugs such as Remdesivir and Chloroquine to prevent the COVID-19 infection. However, these drugs had no impact on curing COVID-19 infection.

Luo et al. [30] proposed the use of the Chinese medicines (CM) containing herbs like radix-astragali, radix-glycyrrhiza, radix-saposhnikoviae, Fructus Forsythia, Lonicerae Japonicae Flos and Rhizoma Atractylodis Macrocephalae to treat COVID-19. These herbs were found effective against SARS and H1N1 Influenza. Evidence from clinical trials, surveys or other population tests suggests that $\mathrm{CM}$ are effective to deter infectious respiratory virus diseases. The analysis showed that the CM Category had smaller side effect than the Non-CM Category (Relative Chance 0.36).

Chen et al. [7] suggested a treatment plan against COVID-19 based on the transplantation of Mesenchymal Stem Cells (MSCs), which was implemented successfully for the treatment of Virus-Induced Pneumonia and H7N9Induced ARDS in 2013. 44 Patients with H7N9-induced ARDS were segregated as a Control Group and 17 patients with H7N9-induced ARDS served as an experimental community of Allogeneic Menstrual-Blood-derived MSCs. It was notable that MSCs transplantation greatly decreased the death rate of the experimental community (17.6\%) compared to the control group (54.5\%). Since H7N9 and COVID-19 share similar symptoms resulting in multi-organ failure, MSC-based treatment could be a possible alternative for COVID-19 treatment.

Shereen et al. [40] analysed the development and pathogenicity of COVID-19 and the prior human coronaviruses-SARS and MERS. They also reviewed the approaches for developing effective vaccines and therapeutic combinations to cope with this viral outbreak. Yuli et al. [45] highlighted the clinical research performed by Dr. Sulianti Saroso, Infectious Disease Hospital, utilizing biochemical assays. The biological samples were obtained from 13 patients with MERS-CoV infection. Viral RNA was insulated and transformed to C-DNA, and used as the guide to identify 12 viral panels based on traditional PCR and sequencing. Viral etiological agents found in patients were Human Metapneumovirus, Enterovirus D68, Rhinovirus C, HCoV 229E, Herpes Simplex Virus Type 1, H1N1, H3N2, Dengue Virus and Rhinovirus A60. The genomes of nine viral agents within various taxa were identified in the MERS-CoV patients, including human metapneumovirus, influenza virus, coronavirus, herpesvirus enterovirus, and paramyxovirus. This study highlights the need for a detailed examination to encounter other secondary viruses that cause more damage than COVID-19. By this method, the mortality rate of COVID19 can be controlled by treating the secondary catalyst viruses responsible for demises.

Dong et al. [13] addressed the possible effects of drugs such as Favipiravir, Chloroquine, Remdesivir, and Arbidol against SARS-CoV-2 and successful results were obtained. $\mathrm{Su}$ et al. [42]. discussed the age as a factor of recovery. SARS-Cov-2 can be shed in the stool of the patients in the recovery phase. It was observed adults have shorter shedding time than children and aged people. They analysed the possible causes of this finding and recommend that a negative stool sample should be included in a patient's discharge criteria.

Metcalf [33] developed a new approach to improve biological resistance of the patient using stem cells against COVID-19. They discussed how synthetic stem cell can help in reducing the mortality rate due to COVID-19.

Zhang and Liu [46] discussed the alternative treatments for COVID-19 since no vaccine is currently available in market. They performed an online search on Coronovirus and RNA Virus therapy and observed that general therapies, Coronavirus-specific therapies, and antibiotic therapies would be useful in the battle against COVID-19. They also suggested that infected patient's nutritious standing should be evaluated before general treatments. The studies on the administration of Influenza vaccine and convalescent serum against COVID-19 need attention.

Daw et al. [10] and Chen et al. [8] advocated that the patients were the key cause of COVID-19 infection. They described the benefits of dynamic surveillance of SARSCoV-2 RNA in the recorded case of COVID-19. The SARS-Cov-2 RNA Oropharyngeal Swab Test was recommended as a discharge criterion for recovered patients. Since the number of discharged patients is escalating, the hospitals will not be equipped for handling the asymptomatic discharged patients with COVID-19 who were retested positive for SARS-CoV-2. In such cases, Zhang et al. [48] advocated the need for self-quarantine among the discharged COVID-19 patients.

\section{Concluding remarks}

In this work, a SLR and a comprehensive analysis of 38 articles on COVID-19 are conducted to answer the formulated research questions. The developed $\mathrm{CON}$ analysis determines the three major research themes in the COVID 
literature as (1) the origin, symptoms and modes of transmission of COVID-19, (2) intervention and mitigation strategies against the spread of COVID-19 and traumatization among the public and (3) drugs and treatment plans of COVID-19.

Our comprehensive analysis reveals that COVID-19 originated from the bats and transferred from Wuhan meat market, Hubei province, China through zoonotic mode of transmission. The epidemiological investigation confirms that COVID-19 involves both the asymptomatic and symptomatic transmission with a communicable period of fewer than 3 weeks. The common symptoms include shortness of breath, fever, sputum production, dry cough, sore throat, fatigue, nausea/vomiting, muscle or joint pain, nasal congestion, headache, chills, and diarrhoea. The intervention and mitigation strategies implemented by the governments against the spread of COVID-19 are city lockdown, border closing, strengthening the surveillance to identify the imported COVID-19 cases from other countries, establishing adequate medical team on a rotation basis, reducing patient attendance in hospitals by rescheduling non-critical surgeries, providing e-health care to public, suspicious accidents monitoring, contract tracking and improved airport security hand hygiene, appropriate use of personal protective equipment, universal masking, and creating transparency and public access to disease data using maps/cartograms. For eradication of the COVID-19, the following drugs and treatment plans are proposed in the literature. Nutrition and immunity enhancers such as Omega-3 polyunsaturated fatty acids (PUFA), Zinc, Vitamin A and C, Chinese medicine, etc., and treatment plans such as Arbidol, Lopinavir/Ritonavir, Convalescent plasma and Mesenchymal Stem Cells and drugs including Remdesivir, Hydroxychloroquine, azithromycin and Favipiravir are analysed. The development of COVID-19 cured people's RNA-virus related vaccines and avian infectious bronchitis (IBV) vaccine are still under study. We conclude by stating that the current work can serve as an aid to health-care researchers in developing the anti-viral vaccine against COVID-19 and helps the administrators in controlling the spread of COVID-19.

\section{References}

1. Ammad M, Din U, Krishna L, Boppana T. 2020;000:6553 (in press).

2. Banerjee D. The COVID-19 outbreak: crucial role the psychiatrists can play. Asian J Psychiatr. 2020;50:102014.

3. Bastian M, Heymann S, Jacomy M. Gephi: an open source software for exploring and manipulating networks. In: Third international AAAI conference on weblogs and social media. 2009;361-62. https://doi.org/10.1136/qshc.2004.010033.
4. Bernard Stoecklin S, Rolland P, Silue Y, et al. First cases of coronavirus disease 2019 (COVID-19) in France: surveillance, investigations and control measures, January 2020. Euro Surveill. 2020;25(6):2000094. https://doi.org/10.2807/1560-7917.es.2020. 25.6.2000094.

5. Callon M, Courtial JP, Turner WA, Bauin S. From translations to problematic networks: an introduction to co-word analysis. Soc Sci Inf. 1983;22(2):191-235. https://doi.org/10.1177/ 053901883022002003.

6. Catton H. Global challenges in health and health care for nurses and midwives everywhere. Int Nurs Rev. 2020;67(1):4-6. https:// doi.org/10.1111/inr.12578.

7. Chen J, Hu C, Chen L, et al. Clinical study of mesenchymal stem cell treatment for acute respiratory distress syndrome induced by epidemic influenza A (H7N9) infection : a hint for COVID-19 treatment. Engineering. 2020. https://doi.org/10.1016/j.eng.2020. 02.006 .

8. Chen D, Xu W, Lei Z, et al. Recurrence of positive SARS-CoV-2 RNA in COVID-19: a case report. Int $\mathrm{J}$ Infect Dis. 2020;93:297-9.

9. Combe D, Largeron C, De Lyon U. A comparative study of social network analysis tools. Int Work Web Intell Virtual Enterp. 2010;2012(2):1-12.

10. Daw MA, Medicine T, Disease I. Preliminary epidemiological analysis of suspected cases of corona virus Infection in Libya. Travel Med Infect Dis. 2020. https://doi.org/10.1016/j.tmaid. 2020.101634.

11. Deng L, Li C, Zeng Q, Liu X, Li X, Zhang H, Hong Z, Xia J. Arbidol combined with LPV/r versus LPV/r alone against Corona Virus Disease 2019: A retrospective cohort study. J Infect. 2020. https://doi.org/10.1016/j.jinf.2020.03.002

12. de Groot RJ, Baker SC, Baric RS, et al. Middle east respiratory syndrome coronavirus (MERS-CoV): announcement of the coronavirus study group. J Virol. 2013;87(14):7790-2. https:// doi.org/10.1128/jvi.01244-13.

13. Dong L, Hu S, Gao J. Discovering drugs to treat coronavirus disease 2019 (COVID-19). Drug Discov Ther. 2020;14(1):58-60. https://doi.org/10.5582/ddt.2020.01012.

14. Gao P, Zhang H, Wu Z, Wang J. Visualising the expansion and spread of coronavirus disease 2019 by cartograms. Environ Plan A Econ Sp. 2020. https://doi.org/10.1177/0308518x20910162.

15. Haider N, Yavlinsky A, Simons D, et al. Passengers' destinations from China: low risk of Novel Coronavirus (2019-nCoV) transmission into Africa and South America. Epidemiol Infect. 2020;148(February):e41. S0950268820000424.

16. Hoffmann M, Kleine-Weber H, Schroeder S, et al. SARS-CoV-2 cell entry depends on ACE2 and TMPRSS2 and is blocked by a clinically proven protease inhibitor. Cell. 2020. https://doi.org/10. 1016/j.cell.2020.02.052.

17. Hou H, Kretschmer H, Liu Z. The structure of scientific collaboration networks in scientometrics. Scientometrics. 2008;75(2):189-202. https://doi.org/10.1007/s11192-007-1771-3.

18. Hu Z, Song C, Xu C, et al. Clinical characteristics of 24 asymptomatic infections with COVID-19 screened among close contacts in Nanjing, China. Sci China Life Sci. 2020. https://doi. org/10.1007/s11427-020-1661-4.

19. Info A. Infection, genetics and evolution letter to editor. 2020;82(March):1-3.

20. Jalava K. First respiratory transmitted food borne outbreak? Int J Hyg Environ Health. 2020;226(February):113490.

21. Kim JY, Ko JH, Kim Y, et al. Viral load kinetics of SARS-CoV-2 infection in first two patients in Korea. J Korean Med Sci. 2020;35(7):1-7. https://doi.org/10.3346/jkms.2020.35.e86.

22. Lai C, Hung Y, Wang C. Asymptomatic carrier state, acute respiratory disease, and pneumonia due to severe acute respiratory 
syndrome coronavirus 2 (SARS-CoV-2): facts and myths. J Microbiol Immunol Infect. 2020;2:10. https://doi.org/10.1016/j.jmii. 2020.02.012.

23. Lancichinetti A, Fortunato S. Limits of modularity maximization in community detection. Phys Rev E Stat Nonlinear Soft Matter Phys. 2011;84(6):1-8. https://doi.org/10.1103/PhysRevE.84. 066122.

24. Lee PC, Su HNN. Investigating the structure of regional innovation system research through keyword co-occurrence and social network analysis. Innov Manag Policy Pract. 2010;12(1):26-40. https://doi.org/10.5172/impp.12.1.26.

25. Li Q, Feng W. Trend and forecasting of the COVID-19 outbreak in China. 2020. https://doi.org/10.1016/j.jinf.2020.02.014.

26. Li Z, Ge J, Yang M, et al. Vicarious traumatization in the general public, members, and non-members of medical teams aiding in COVID-19 control. Brain Behav Immun. 2020. https://doi.org/10. 1016/j.bbi.2020.03.007.

27. Lim J, Jeon S, Shin HY, Kim MJ, Seong YM, Lee WJ, Choe KW, Kang YM, Lee B, Park SJ. Case of the index patient who caused tertiary transmission of coronavirus disease 2019 in Korea: the application of lopinavir/ritonavir for the treatment of COVID-19 pneumonia monitored by quantitative RT-PCR. J Korean Med Sci. 2020;35(7):1-6. https://doi.org/10.3346/jkms.2020.35.e88.

28. Log S. Understanding SARS-CoV-2 and the 1/9. 2020:1-9.

29. Luan J, Lu Y, Jin X, Zhang L. Spike protein recognition of mammalian ACE2 predicts the host range and an optimized ACE2 for SARS-CoV-2 infection. Biochem Biophys Res Commun. 2020;526:165-9.

30. Luo H, Tang Q, Shang Y, et al. Can Chinese medicine be used for prevention of corona virus disease 2019 (COVID-19)? A review of historical classics, research evidence and current prevention programs. Chin J Integr Med. 2020. https://doi.org/10.1007/ s11655-020-3192-6.

31. Maier HJ, Walker JM. Coronaviruses IN Series Editor.

32. Max Roser HR, Ortiz-Ospina E. Coronavirus disease (COVID19)? Statistics and research. Our World in Data 2020.

33. Metcalfe SM. Mesenchymal stem cells and management of COVID-19 pneumonia. Med Drug Discov. 2020;5:100019.

34. Newman MEJ, Girvan M. Finding and evaluating community structure in networks. Phys Rev E Stat Nonlinear Soft Matter Phys. 2004;69(22):1-15. https://doi.org/10.1103/physreve.69. 026113.

35. Rohde RE. Novel coronavirus (2019-nCoV) update: uncoating the virus. Am Soc Microbiol. 2020;(Cdc). https://asm.org/Arti cles/2020/January/2019-Novel-Coronavirus-2019-nCoV-UpdateUncoating.

36. Paraskevis D, Kostaki EG, Magiorkinis G, Panayiotakopoulos G, Sourvinos G. Full-genome evolutionary analysis of the novel corona virus (2019-nCoV) rejects the hypothesis of emergence as a result of a recent recombination event. Infect Genet Evol. 2020;79(January):104212.

37. Qin C, Liu F, Yen TC, Lan X. 18 F-FDG PET/CT findings of COVID-19: a series of four highly suspected cases. Eur J Nucl
Med Mol Imaging. 2020. https://doi.org/10.1007/s00259-02004734-w.

38. Rajagopal V, Prasanna Venkatesan S, Goh M. Decision-making models for supply chain risk mitigation: a review. Comput Ind Eng. 2017;113(January):646-82. https://doi.org/10.1016/j.cie. 2017.09.043.

39. Sexton NR, Smith EC, Blanc H, Vignuzzi M, Peersen OB, Denison MR. Homology-based identification of a mutation in the coronavirus RNA-dependent RNA polymerase that confers resistance to multiple mutagens. J Virol. 2016;90(16):7415-28. https://doi.org/10.1128/jvi.00080-16.

40. Shereen MA, Khan S, Kazmi A, Bashir N, Siddique R. COVID19 infection: origin, transmission, and characteristics of human coronaviruses. J Adv Res. 2020. https://doi.org/10.1016/j.jare. 2020.03.005.

41. Song $\mathrm{Z}, \mathrm{Xu} \mathrm{Y}, \mathrm{Bao} \mathrm{L}$, et al. From SARS to MERS, thrusting coronaviruses into the spotlight. Viruses. 2019. https://doi.org/10. 3390/v11010059.

42. Su L, Zhang Y, Zhang X, Gai Z, Zhang Z. Do children need a longer time to shed SARS-CoV-2 in stool than adults? J Microbiol Immunol Infect. 2020. https://doi.org/10.1016/j.jmii.2020.03. 010 .

43. Wind TR, Rijkeboer M, Andersson G. The COVID-19 pandemic: The 'black swan' for mental health care and a turning point for e-health. Int Interv. 2020;20:100317.

44. Yan Z, Spaulding HR, Biology R, et al. YouTube as source of information on 2019 novel coronavirus outbreak: a cross sectional study of English and Mandarin Content. Travel Med Infect Dis. 2020;10:10. https://doi.org/10.1016/j.tmaid.2020.101636.

45. Yuli T, Wiyatno A, Sarry T, et al. Detection of multiple viral sequences in the respiratory tract samples of suspected Middle East respiratory syndrome coronavirus patients in Jakarta, Indonesia 2015-2016. Int J Infect Dis. 2015;2019(86):102-7.

46. Zhang L, Liu Y. Potential interventions for novel coronavirus in china: a systematic review. New York: Wiley; 2020. https://doi. org/10.1002/jmv.25707.

47. Zhang C, Zheng W, Huang X, Bell EW, Zhou X, Zhang Y. Protein structure and sequence re-analysis of 2019-nCoV genome does not indicate snakes as its intermediate host or the unique similarity between its spike protein insertions and HIV-1. J Proteome Res. 2020. https://doi.org/10.1021/acs.jproteome.0c00129.

48. Zhang Y, Yu L, Tang L, et al. A promising anti-cytokine-storm targeted therapy for COVID-19: the artificial-liver blood-purification system. Engineering. 2020. https://doi.org/10.1016/j.eng. 2020.03.006.

49. Zhou P, Yang X-L, Wang X-G, et al. A pneumonia outbreak associated with a new coronavirus of probable bat origin. Nature. 2020;579(7798):270-3. https://doi.org/10.1038/s41586-0202012-7.

Publisher's Note Springer Nature remains neutral with regard to jurisdictional claims in published maps and institutional affiliations. 